\title{
On Edge Irregular Reflexive Labeling for Generalized Prism
}

\author{
Chenxi Wang, ${ }^{1}$ M. J. A. Khan, ${ }^{2}$ M. Ibrahim $\mathbb{D}^{,}{ }^{2}$ E. Bonyah $\mathbb{D},{ }^{3}$ M. K. Siddiqui, ${ }^{4}$ and S. Khalid ${ }^{4}$ \\ ${ }^{1}$ Alliance Manchester Business School, The University of Manchester, Manchester, UK \\ ${ }^{2}$ Centre for Advanced Studies in Pure and Applied Mathematics, Bahauddin Zakariya University, Multan, Pakistan \\ ${ }^{3}$ Department of Mathematics Education, Akenten Appiah-Menka University of Skills Training and Entrepreneurial Development, \\ Kumasi 00233, Ghana \\ ${ }^{4}$ Department of Mathematics, Comsats University Islamabad, Lahore Campus, Lahore, Pakistan
}

Correspondence should be addressed to E. Bonyah; ebonyah@aamusted.edu.gh

Received 3 January 2022; Accepted 26 January 2022; Published 7 March 2022

Academic Editor: M. T. Rahim

Copyright (C) 2022 Chenxi Wang et al. This is an open access article distributed under the Creative Commons Attribution License, which permits unrestricted use, distribution, and reproduction in any medium, provided the original work is properly cited.

Among the various ideas that appear while studying graph theory, which has gained much attraction especially in graph labeling, labeling of graphs gives mathematical models which value for a vast range of applications in high technology (data security, cryptography, various problems of coding theory, astronomy, data security, telecommunication networks, etc.). A graph label is a designation of graph elements, i.e., the edges and/or vertex of a group of numbers (natural numbers), and is called assignment or labeling. The vertex or edge labeling is related to their domain asset of vertices or edges. Likewise, for total labeling, we take the domain as vertices and edges both at the same time. The reflexive edge irregularity strength (res) is total labeling in which weights of edges are not the same for all edges and the weight of an edge is taken as the sum of the edge labels and the vertices associated with that edge. In the res, the vertices are labeled with nonnegative even integers while the edges are labeled with positive integers. We have to make the labels minimum, whether they are associated with vertices or edges. If such labeling exists, then it is called the res of $H$ and is represented as $s$ res $(H)$. In this paper, we have computed the res for the Cartesian product of path and cycle graph which is also known as generalizing prism.

\section{Introduction}

Any graph $H$ is the combination of vertices $V(H)$ along with a possibly nonempty edge set $E(H)$ of 2-element subsets of $V(H)$. In this paper, all the chosen graphs are finite, without direction, nontrivial, connected, and simple (without loops and multiedges). For details about notations, see [1, 2]. Nonnegative integers are used in this research. In 1988, Chartrand et al. [3] proposed the labeling problems in graph theory. Assign the edges positive integer to all connected simple graphs such as the graph became irregular. The irregular labeling is defined as $\psi: E(H) \longrightarrow\{1,2,3, \ldots, m\}$ and is called irregular $m$-labeling for graph $H$ if all the separate nodes $u$ and $u^{\prime}$ have distinctly weights, that is,

$$
\sum_{x \in V} \psi(u x) \neq \sum_{y \in V} \psi\left(u^{\prime} y\right)
$$

Lahel, in [4], studied, in detail, for the irregularity strength. For more results, see the works of Nierhoff in [5], Dimitz et al. in [6], Amar and Togni in [7], and Gyarfas in [8].

In [9], A. Ahmad et al. defined on edge irregularity strength $(e s(H))$ for any two edges $u_{1} u_{2}$ and $u_{1}^{\prime} u_{2}^{\prime}$ that the weights $w_{\phi}\left(u_{1} u_{2}\right)$ and $w_{\phi}\left(u_{1}^{\prime} u_{2}^{\prime}\right)$ are distinct, as weight for an edge $u_{1} u_{2} \in E(H)$ is $w_{\phi}\left(u_{1} u_{2}\right)=\phi\left(u_{1}\right)+\phi\left(u_{2}\right)$.

In [10], Bača et al. defined the parameter of total labeling for edge as well as vertex of graph and found the weights of an edge as sum of three integers which include the edge label and the labels of two vertices associated with that edge, and finally, every edge has distinct weight. For detailed studies on total edge irregularity strength, see $[10,11]$.

The concept of total edge irregularity strength has been generalized by Zhang et al. in [12] for graph will be reflexive edge irregularity strength $m$-labeling. 
If, for any graph $H$, the total $m$-labeling defined the mapping $\psi_{e^{\prime}}: E(H) \longrightarrow\left\{1,2,3 \ldots, m_{e^{\prime}}\right\}$ and $\psi_{v^{\prime}}: V(H)$ $\longrightarrow\left\{0,2,4, \ldots, 2 m_{v^{\prime}}\right\}$, the mapping $\psi$ is a total $m$-mapping of $H$ such that $\psi(a)=\psi_{v}(a)$ if $a \in V(H)$ and $\psi(a)=\psi_{e}(a)$ if $a \in E(H)$, where $k=\max \left\{m_{e^{\prime}}, 2 m_{v^{\prime}}\right\}$.

The total $p$-labeling $\psi$ will be edge irregular reflexive $p$-labeling of the graph $H$ if, for all the different edges say $u_{1} u_{2}$ and $u_{1}^{\prime} u_{2}^{\prime}$, the weights $w_{\phi}\left(u_{1} u_{2}\right)$ and $w_{\phi}\left(u_{1}^{\prime} u_{2}^{\prime}\right)$ are not the same for every choice of edges where the weight for any edge suppose $u_{1} u_{2} \in E(H)$ is $w_{\phi}\left(u_{1} u_{2}\right)=\phi\left(u_{1}\right)$ $+\phi\left(u_{1} u_{2}\right)+\phi\left(u_{2}\right)$.

The smallest value of $p$ for which such mapping exists is said to be res of the graph $H$ and is represented by res $(H)$. For details in reflexive edge irregularity strength, see [13-17].

For res $(H)$, Nierhoff [5] proposed that for any graph $H(s, t)$ with maximum degree $\Delta(H)$ satisfies

$$
\operatorname{res}(H)=\max \left\{\left\lceil\frac{|t|}{3}+r\right\rceil,\left\lfloor\frac{\Delta}{2}+1\right\rfloor\right\},
$$

where $r$ will be 1 for $|t| \equiv 2,3(\bmod 6)$; it will be 0 , otherwise.

In [12], the lemma is proven.

Lemma 1. For all graph say $H$,

$$
\operatorname{res}(H) \geq \begin{cases}\left\lceil\frac{|t|}{3}\right\rceil+1, & \text { if }|t| \equiv 2,3(\bmod 6), \\ \left\lceil\frac{|t|}{3}\right\rceil, & \text { if }|t| \equiv 1,4,5(\bmod 6) .\end{cases}
$$

In the present research paper, we have investigated the res for the Cartesian product of paths and cycles.

1.1. Definition. The Cartesian product $P$ and $Q$ graphs is represented as $P \square Q$ and is the graph with vertices set $V(P) \times V(Q)$, with vertices $\left(u_{1}, u_{1}^{\prime}\right)$ and $\left(w_{1}, w_{1}^{\prime}\right)$ will be adjacent if and only if $u_{1}=w_{1}$ and $u_{1}^{\prime} w_{1}^{\prime} \in E(Q)$ or $u_{1}^{\prime}=w_{1}^{\prime}$ and $u_{1} w_{1} \in E(P)$.

Theorem 1. Let $P_{d}$ and $C_{c}$ be path and cycle, respectively; then, for edge irregular reflexive strength of $P_{d} \square C_{c}$ with $d \geq 3$ and $c \geq 2$. We have

$\operatorname{res}\left(P_{d} \square C_{c}\right)= \begin{cases}\left\lceil\frac{(2 d-1) c}{3}\right\rceil+1, & \text { if }|(2 d-1) c| \equiv 2,3(\bmod 6), \\ \left\lceil\frac{(2 d-1) c}{3}\right\rceil, & \text { if }|(2 d-1) c| \equiv 1,4,5,6(\bmod 6) .\end{cases}$

Proof. As $P_{d} \square C_{c}$ has $(2 d-1) c$ edges, therefore, from Lemma 1 , we obtain

$\operatorname{res}\left(P_{d} \square C_{c}\right) \geq \begin{cases}\left\lceil\frac{(2 d-1) c}{3}\right\rceil+1, & \text { if }|(2 d-1) c| \equiv 2,3(\bmod 6), \\ \left\lceil\frac{(2 d-1) c}{3}\right\rceil, & \text { if }|(2 d-1) c| \equiv 1,4,5,6(\bmod 6) .\end{cases}$
Next, we will show that

$\operatorname{res}\left(P_{d} \square C_{c}\right) \leq \begin{cases}\left\lceil\frac{(2 d-1) c}{3}\right\rceil+1, & \text { if }|(2 d-1) c| \equiv 2,3(\bmod 6), \\ \left\lceil\frac{(2 d-1) c}{3}\right\rceil, & \text { if }|(2 d-1) c| \equiv 1,4,5,6(\bmod 6) .\end{cases}$

We defined a $f$-labeling for this on $\left(P_{d} \square C_{c}\right)$ as follows: $\forall 1 \leq j \leq c$.

Let $e=x_{i, j}, h=x_{i+1, j}$, and $k=x_{i, j+1}$

Case 1. When $d \equiv 0(\bmod 3), c$ is odd:

$$
f(e)= \begin{cases}c(i-1), & \text { for } 1 \leq i \leq \frac{2 d-3}{3}(i \text { is odd }), \\ c(i-1)-1, & \text { for } 2 \leq i \leq \frac{2 d}{3}(i \text { is even }), \\ k, & \text { for } \frac{2 d+3}{3} \leq i \leq d .\end{cases}
$$

When $c \equiv 1(\bmod 6)$,

$$
f((e)(k))= \begin{cases}j, & \text { for } 1 \leq i \leq \frac{2 d-3}{3}, \\ j+2, & \text { for } 2 \leq i \leq \frac{2 d}{3}, \\ \frac{6 c i-2(2 c d-c-1)}{3}+j, & \text { for } \frac{2 d+3}{3} \leq i \leq d,\end{cases}
$$$$
f((e)(h))= \begin{cases}j+1, & \text { for } 1 \leq i \leq \frac{2 d-3}{3} \\ \frac{c-1}{3}+1+j & \text { for } i=\frac{2 d}{3} \\ \frac{6 c i-(4 c d+c+2)}{3}+j, & \text { for } \frac{2 d+3}{3} \leq i \leq d-1 .\end{cases}
$$

When $c \equiv 3(\bmod 6)$,

$$
\begin{aligned}
& f((e)(k))= \begin{cases}j, & \text { for } 1 \leq i \leq \frac{2 d-3}{3}, \\
j+2, & \text { for } 2 \leq i \leq \frac{2 d}{3}, \\
\frac{6 c i-2(2 c d+2 c+3)}{3}+j & \text { for } \frac{2 d}{3}+1 \leq i \leq d,\end{cases} \\
& f((e)(h))= \begin{cases}j+1, & \text { for } 1 \leq i \leq \frac{2 d-3}{3}, \\
\frac{2(c-1)}{3}+1+j & \text { for } i=\frac{2 d}{3}, \\
\frac{6 c i-4 c(d+1)}{3}+j, & \text { for } \frac{2 d+3}{3} \leq i \leq d-1 .\end{cases}
\end{aligned}
$$

When $c \equiv 5(\bmod 6)$, 


$$
\begin{aligned}
& f((e)(k))= \begin{cases}j, & \text { for } \left.1 \leq i \leq \frac{2 d-3}{3} \text { ( } i \text { is odd }\right), \\
j+1, & \text { for } 2 \leq i \leq \frac{2 d}{3}(i \text { is even }), \\
\frac{6 c i-2(2 c d+2 d-1)}{3}+j, & \text { for } \frac{2 d+3}{3} \leq i \leq d,\end{cases} \\
& f((e)(h))= \begin{cases}j+1, & \text { for } 1 \leq i \leq \frac{2 d}{3}-1, \\
\left.\frac{c-5}{3}\right)+3+j & \text { for } i=\frac{2 d}{3}, \\
\frac{6 c i-(4 c d+c-2)}{3}+j, & \text { for } \frac{2 d}{3}+1 \leq i \leq d-1 .\end{cases}
\end{aligned}
$$

Case 2. When $d \equiv 0(\bmod 3), c$ is even:

$$
f(e)= \begin{cases}d(i-1), & \text { for } 1 \leq i \leq \frac{2 d}{3} \\ k, & \text { for } \frac{2 d+3}{3} \leq i \leq d .\end{cases}
$$

When $d \equiv 0(\bmod 6)$,

$$
\begin{aligned}
& f((e)(k))= \begin{cases}j, & \text { for } 1 \leq i \leq \frac{2 d}{3}, \\
(6 i-4 d-4) \frac{c}{3}+j, & \text { for } \frac{2 d+3}{3} \leq i \leq d,\end{cases} \\
& f((e)(h))= \begin{cases}j, & \text { for } 1 \leq i \leq \frac{2 d}{3}-1, \\
\frac{c}{3}+j & \text { for } i=\frac{2 d}{3}, \\
(6 i-4 d-1) \frac{c}{3}+j, & \text { for } \frac{2 d}{3}+1 \leq i \leq d-1 .\end{cases}
\end{aligned}
$$

When $c \equiv 2(\bmod 6)$,

$$
\begin{aligned}
& f((e)(k))= \begin{cases}j, & \text { for } 1 \leq i \leq \frac{2 d}{3}, \\
\frac{6 c i-4(c d+c+1)}{3}+j, & \text { for } \frac{2 d+3}{3} \leq i \leq d,\end{cases} \\
& f((e)(h))= \begin{cases}j, & \text { for } 1 \leq i \leq \frac{2 d-3}{3}, \\
\left(\frac{c-2}{3}\right)+j & \text { for } i=\frac{2 d}{3}, \\
\frac{6 c i-(4 c d+c+4)}{3}+j, & \text { for } \frac{2 d}{3}+1 \leq i \leq d-1 .\end{cases}
\end{aligned}
$$

When $c \equiv 4(\bmod 6)$,

$$
\begin{aligned}
& f((e)(k))= \begin{cases}j, & \text { for } 1 \leq i \leq \frac{2 d}{3}, \\
\frac{6 c i-4(c d+c+2)}{3}+j, & \text { for } \frac{2 d}{3}+1 \leq i \leq d,\end{cases} \\
& f((e)(h))= \begin{cases}j, & \text { for } 1 \leq i \leq \frac{2 d}{3}-1, \\
\frac{2(c-4)}{3}+j & \text { for } i=\frac{2 d}{3}, \\
\frac{6 c i-(4 c d+c+8)}{3}+j, & \text { for } \frac{2 d}{3}+1 \leq i \leq d-1 .\end{cases}
\end{aligned}
$$

Case 3. When $d \equiv 1(\bmod 3), c$ is even:

$$
f(e)= \begin{cases}c(i-1), & \text { for } 1 \leq i \leq \frac{2 d+1}{3}, \\ k, & \text { for } 2\left(\frac{d+2}{3}\right) \leq i \leq d .\end{cases}
$$

When $d \equiv 1(\bmod 3)$ and $c \equiv 0(\bmod 6)$,

$$
\begin{aligned}
& f((e)(k))= \begin{cases}j, & \text { for } 1 \leq i \leq \frac{2 d+1}{3}, \\
\frac{c(6 i-4 d-4)}{3}+j, & \text { for } \frac{2(d+2)}{3} \leq i \leq d,\end{cases} \\
& f((e)(h))= \begin{cases}j, & \text { for } 1 \leq i \leq 2\left(\frac{d-1}{3}\right), \\
\frac{2 c}{3}+j, & \text { for } i=\frac{2 d+1}{3}, \\
\frac{c(6 i-4 d-1)}{3}+j, & \text { for } \frac{2 d+4}{3} \leq i \leq d-1 .\end{cases}
\end{aligned}
$$

When $d \equiv 1(\bmod 3)$ and $c \equiv 2(\bmod 6)$,

$$
\begin{aligned}
& f((e)(k))= \begin{cases}j, & \text { for } 1 \leq i \leq \frac{2 d+1}{3}, \\
\frac{6 c i-4(c d+c+2)}{3}+j, & \text { for } 2\left(\frac{d+2}{3}\right) \leq i \leq d,\end{cases} \\
& f((e)(h))= \begin{cases}j, & \text { for } 1 \leq i \leq 2\left(\frac{d-1}{3}\right), \\
\frac{2(c-2)}{3}+j, & \text { for } i=\frac{2 d+1}{3}, \\
\frac{6 c i-(4 c d+c+8)}{3}+j, & \text { for } 2\left(\frac{d+2}{3}\right) \leq i \leq d-1 .\end{cases}
\end{aligned}
$$

When $d \equiv 1(\bmod 3)$ and $c \equiv 4(\bmod 6)$, 


$$
\begin{aligned}
& f((e)(k))= \begin{cases}j, & \text { for } 1 \leq i \leq \frac{2 d+1}{3}, \\
\frac{6 c i-4(c d+c+1)}{3}+j & \text { for } 2\left(\frac{d+2}{3}\right) \leq i \leq d,\end{cases} \\
& f((e)(h))= \begin{cases}j, & \text { for } 1 \leq i \leq 2\left(\frac{d-1}{3}\right) \\
\frac{2(c-4)}{3}+2+j, & \text { for } i=\frac{2 d+1}{3}, \\
\frac{6 c i-(4 c d+c+4)}{3}+j, & \text { for } 2\left(\frac{d+2}{3}\right) \leq i \leq d-1 .\end{cases}
\end{aligned}
$$

$$
\begin{aligned}
& f((e)(k))= \begin{cases}j, & \text { for } 1 \leq i \leq \frac{2 d+1}{3}(i \text { is odd }), \\
j+2, & \text { for } 2 \leq i \leq \frac{2(n-1)}{3}(i \text { is even }), \\
\frac{6 c i-(4 c d+4 c-2)}{3}+j & \text { for } \frac{2(d+2)}{3} \leq i \leq d,\end{cases} \\
& f((e)(h))= \begin{cases}j+1, & \text { for } 1 \leq i \leq \frac{2(d-1)}{3}, \\
\frac{2 c+1}{3}+j, & \text { for } i=\frac{2 d+1}{3}, \\
\frac{6 c i-(4 c d+5)}{3}+j, & \text { for } \frac{2(n+2)}{3} \leq i \leq d-1 .\end{cases}
\end{aligned}
$$

When $d \equiv 1(\bmod 3)$ and $c \equiv 3(\bmod 6)$,

$$
f((e)(k))= \begin{cases}j, & \text { for } 1 \leq i \leq \frac{2 d+1}{3}(i \text { is odd }), \\ j+2, & \text { for } 2 \leq i \leq \frac{2(d-1)}{3}(i \text { is even }), \\ \frac{c(6 i-4 d-4)}{3}-2+j & \text { for } \frac{2(d+2)}{3} \leq i \leq d, \\ f((e)(h))= \begin{cases}j+1, & \text { for } 1 \leq i \leq \frac{2(d-1)}{3}, \\ \frac{2 c}{3}-1+j, & \text { for } i=\frac{2 d+1}{3}, \\ \frac{c(6 i-4 d-1)}{3}-2+j & \end{cases} \end{cases}
$$

When $d \equiv 1(\bmod 3)$ and $c \equiv 5(\bmod 6)$, 


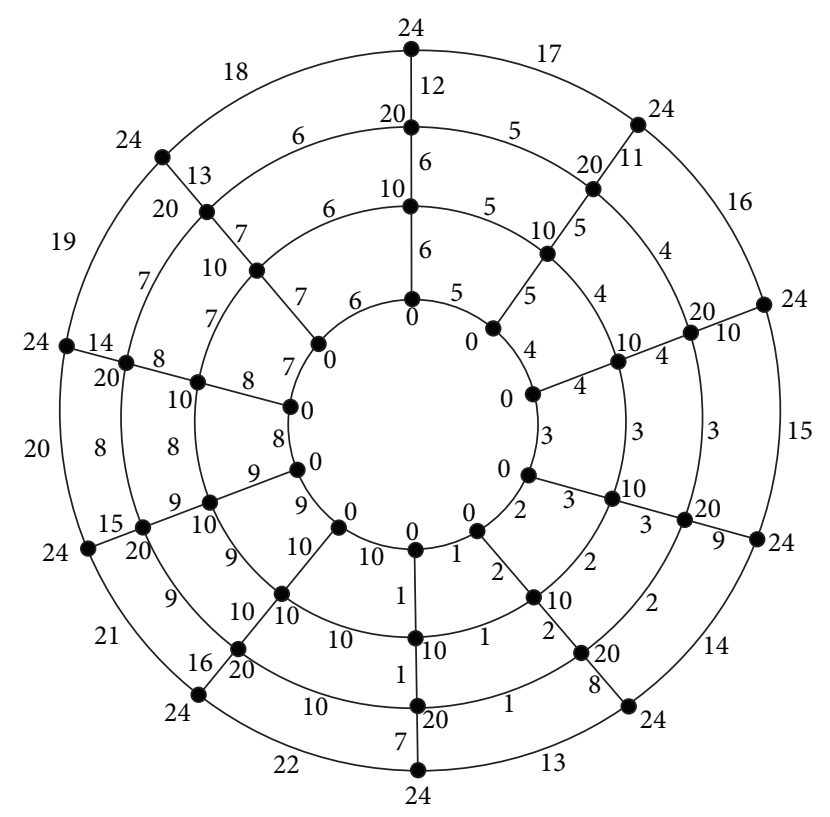

Figure 1: Twenty four labeling of $P_{4} \square C_{10}$.

$$
\begin{aligned}
& f((e)(k))= \begin{cases}j, & \text { for } 1 \leq i \leq \frac{2 d+1}{3}(i \text { is odd }), \\
j+2, & \text { for } 1 \leq i \leq \frac{2(d-1)}{3}(i \text { is even }), \\
\frac{6 c i-4 c(d+1)-2}{3}+j & \text { for } \frac{2(d+2)}{3} \leq i \leq d,\end{cases} \\
& f((e)(h))= \begin{cases}j+1, & \text { for } 1 \leq i \leq 2\left(\frac{d-1}{3}\right) \\
\frac{2(c-5)}{3}+3+j, & \text { for } i=\frac{2 d+1}{3} \\
\frac{6 c i-(4 c d+c+2)}{3}+j, & \text { for } \frac{2(d+2)}{3} \leq i \leq d-1 .\end{cases}
\end{aligned}
$$

Case 5. When $d \equiv 2(\bmod 3), c$ is even and $c \geq 4$ :

$$
\begin{aligned}
& f(e)= \begin{cases}c(i-1), & \text { for } 1 \leq i \leq \frac{2 d-1}{3}, \\
k, & \text { for } \frac{2 d+2}{3} \leq i \leq d,\end{cases} \\
& f((e)(k))= \begin{cases}j, & \text { for } 1 \leq i \leq \frac{2 d+2}{3}, \\
\frac{6 c i-4(c d-2 c+3)}{3}+j & \text { for } \frac{2 d+5}{3}+3 \leq i \leq d,\end{cases}
\end{aligned}
$$




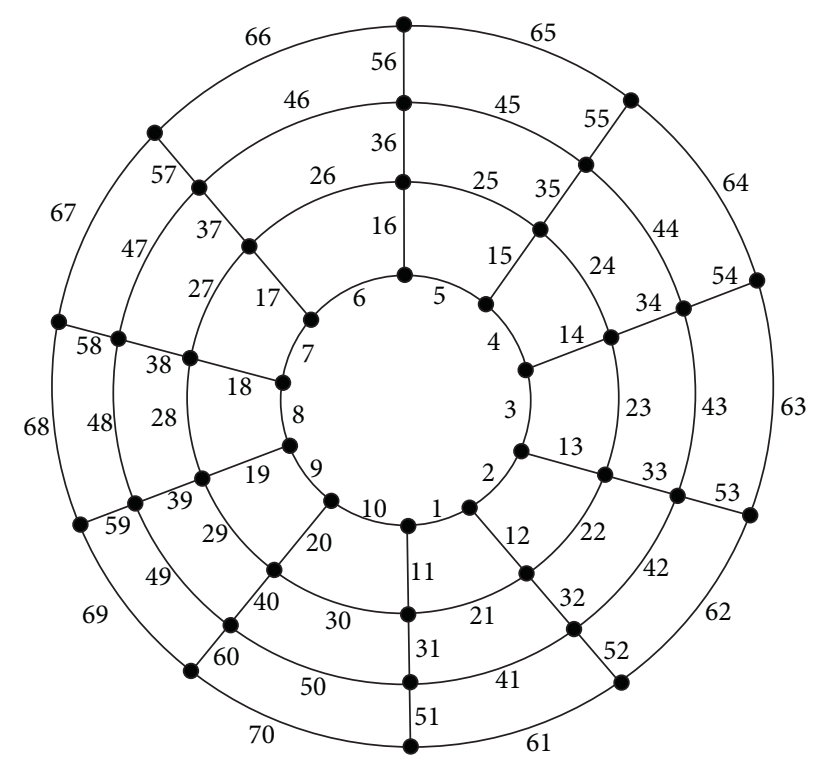

FIgURE 2: Edge weights of $P_{4} \square C_{10}$.

$$
f((e)(h))= \begin{cases}j, & \text { for } 1 \leq i \leq \frac{2 d-1}{3}, \\ \frac{6 c i-4(d-2) c-9}{3}+j, & \text { for } \frac{2 d+2}{3} \leq i \leq d-1 .\end{cases}
$$

Case 6. When $d \equiv 2(\bmod 3), c$ is odd and $c \geq 3$ :

$$
\begin{aligned}
& f(e)= \begin{cases}c(i-1), & \text { for } 1 \leq i \leq \frac{2 d-1}{3}(i \text { is odd }), \\
c(i-1)-1, & \text { for } 2 \leq i \leq \frac{2 d+2}{3}(i \text { is even }), \\
k, & \text { for } \frac{2 d+5}{3} \leq i \leq d,\end{cases} \\
& f((e)(k))= \begin{cases}j, & \text { for } 1 \leq i \leq \frac{2 d-1}{3}(i \text { is odd }), \\
j+2, & \text { for } 2 \leq i \leq \frac{2 d+2}{3}(i \text { is even }), \\
\frac{6 c i-4(d+1) c}{3}-2+j & \text { for } \frac{2 d+5}{3} \leq d,\end{cases} \\
& f((e)(h))= \begin{cases}j+1, & \text { for } 1 \leq i \leq \frac{2 d-1}{3}, \\
(2 c-2) i-\frac{(4 d+1)(c-1)}{3}-1+j, & \text { for } \frac{2 d+2}{3} \leq i \leq d-1 .\end{cases}
\end{aligned}
$$


Weights for reflexive edges is given as follows: for $1 \leq i \leq d-11, \leq i \leq c$, weight of edge $\left(\left(x_{i, j}\right)\left(x_{i+1, j}\right)\right)$ is $(2 i-1) c+j$ and weight of the edge $\left(\left(x_{i, j}\right)\left(x_{i, j+1}\right)\right)$ is $2 c(i-1)+j$,

None of the two edges are of the same weight. So, we get the required result, for $c \geq 2$ and $d \geq 3$, which completes the proof.

\section{Conclusion}

In the present paper, we found the reflexive edge irregularity strength for generalized prism graph $\left(P_{d} \square C_{c}\right)$, for $d \geq 3$ and $c \geq 2$.

\section{Data Availability}

No data were used to support the findings of the study.

\section{Conflicts of Interest}

The authors declare that they have no conflicts of interest.

\section{References}

[1] S. Brandt, J. Miškuf, and D. Rautenbach, "On a conjecture about edge irregular total labelings," Journal of Graph Theory, vol. 57, no. 4, pp. 333-343, 2008.

[2] G. Chartrand, L. Lesniak, and P. Zhang, Graphs and Diagraphs, Taylor and Francis Group Boca Raton, New York, USA, 6th edition, 2016.

[3] G. Chartrand, M. S. Jacobson, J. Lehel, O. R. Oellermann, S. Ruiz, and F. Saba, "Irregular networks," Congressus Numerantium, vol. 64, pp. 187-192, 1988.

[4] J. Lahel, "Facts and quests on degree irregular assignment," in Graph Theory, Combinatorics and Applications, pp. 765-782, Wley, New York, NY, USA, 1991.

[5] T. Nierhoff, "A tight bound on the irregularity strength of graphs," SIAM Journal on Discrete Mathematics, vol. 13, no. 3, pp. 313-323, 2000.

[6] J. H. Dimitz, D. K. Garnick, and A. Gyárfás, "On the irregularity strength of the $m \times n$ grid," Journal of Graph Theory, vol. 16, pp. 355-374, 1992.

[7] D. Amar and O. Togni, "Irregularity strength of trees," Discrete Mathematics, vol. 190, no. 1-3, pp. 15-38, 1998.

[8] A. Gyárfás, "The irregularity strength of $K_{m, m}$ is 4 for odd $m$," Discrete Mathematics, no. 71, pp. 273-274, 1998.

[9] A. Ahmad, O. B. S. Al-Mushayt, and M. Bača, "On edge irregularity strength of graphs," Applied Mathematics and Computation, vol. 243, pp. 607-610, 2014.

[10] M. Baca, M. Miller, and J. Ryan, "On irregular total labellings," Discrete Mathematics, vol. 307, no. 11-12, pp. 1378-1388, 2007.

[11] A. Ahmad, M. Bača, and M. K. Siddiqui, "On edge irregular total labeling of categorical product of two cycles," Theory of Computing Systems, vol. 54, no. 1, pp. 1-12, 2014.

[12] X. Zhang, M. Ibrahim, S. Bokhary, and M. Siddiqui, "Edge irregular reflexive labeling for the disjoint union of gear graphs and prism graphs," Mathematics, vol. 6, no. 9, pp. 142-155, 2018.

[13] I. H. Agustin, M. I. Utoyo, M. Dafik, M. Venkatachalam, and F. Surahmat, "On the construction of the reflexive vertex $k$ labeling of any graph with pendant vertex," International
Journal of Mathematics and Mathematical Sciences, vol. 2020, no. 1, 8 pages, Article ID 7812812, 2020.

[14] M. Baca, M. Irfan, J. Ryan, A. Semanicov-Fenovckov, and D. Tanna, "Note on edge irregular reflexive labelings of graphs," AKCE International Journal of Graphs and Combinatorics, vol. 16, no. 2, pp. 145-157, 2019.

[15] M. Baca, M. Irfan, J. Ryan, A. Semanicov-Fenovckov, and D. Tanna, "On edge irregular reflexive labellings for the generalized friendship graphs," Mathematics, vol. 5, no. 4, pp. 67-77, 2017.

[16] M. Ibrahim, S. Majeed, and M. K. Siddiqui, "Edge irregular reflexive labeling for star and caterpillar graphs," TWMS Journal of Applied and Engineering Mathematics, vol. 10, no. 3, pp. 718-726, 2020.

[17] D. Tanna, J. Ryan, and A. Semabičovă-Feňovčkovă, "A reflexive edge irregular labelings of prisms and wheels," Australasian Journal of Combinatorics, vol. 69, pp. 394-401, 2017. 